Musées, Patrimoine et Culture scientifiques et techniques

$178 \mid 2018$

juillet-août 2018

\title{
Le marché de l'exposition itinérante internationale, ses acteurs et sa filière
}

\section{Méliné Kéloglanian}

\section{OpenEdition \\ Journals}

Édition électronique

URL : http://journals.openedition.org/ocim/2652

DOI : $10.4000 /$ ocim.2652

ISSN : 2108-646X

Éditeur

OCIM

Édition imprimée

Date de publication : 1 juillet 2018

Pagination : $12-18$

ISSN : 0994-1908

Référence électronique

Méliné Kéloglanian, «Le marché de l'exposition itinérante internationale, ses acteurs et sa filière », La Lettre de I'OCIM [En ligne], 178 | 2018, mis en ligne le 01 juillet 2019, consulté le 07 septembre 2019. URL : http://journals.openedition.org/ocim/2652 ; DOI : 10.4000/ocim.2652

Ce document a été généré automatiquement le 7 septembre 2019.

Tous droits réservés 


\section{Le marché de l'exposition itinérante internationale, ses acteurs et sa filière}

Méliné Kéloglanian 
Affiche faisant la publicité du promoteur hongrois Multimedia Kft., parue dans IQ, magazine publié par l'organisation ILMC (International Live Music Community). L'article (The Expo Explosion, IQ, Issue 24, Q2 2009, p. 26) dans lequel figure cette publicité s'interroge sur les raisons ayant poussé un certain nombre d'acteurs de l'industrie de la musique live à se diversifier dans le secteur de l'exposition itinérante internationale.

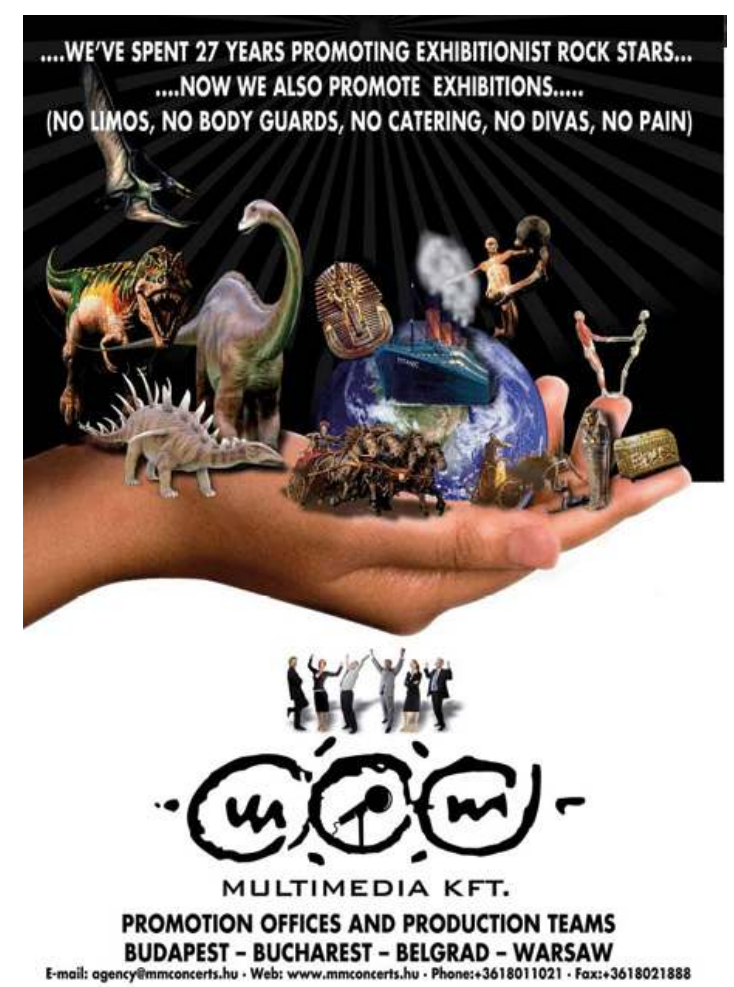

(c) DR

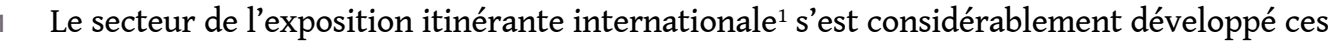
quinze dernières années. Si ces expositions étaient jusque dans le dernier quart du XXe siècle l'apanage des institutions muséales, elles s'en sont pour partie aujourd'hui émancipées. Elles peuvent être désormais organisées et reçues par des types d'acteurs extrêmement variés, parmi lesquels des acteurs privés. Ces derniers, soumis à une logique de rentabilité qui constitue la condition sine qua non de leur existence et de leur pérennité, ont achevé d'installer une lecture mercatique des activités d'itinérance. Il incombe alors au milieu muséal de se donner les moyens de comprendre et de caractériser l'évolution de ce secteur d'activité, sans quoi il ne pourra s'adapter ou réagir à ces nouvelles logiques de fonctionnement.

2 Afin de contribuer à cette caractérisation, nous nous intéresserons à ce secteur dans sa globalité et appréhenderons les manières dont interagissent les différents acteurs y intervenant. Pour ce faire nous considèrerons tout d'abord les motivations des principaux types d'acteurs. Nous examinerons ensuite l'organisation de la filière de l'exposition itinérante internationale à laquelle ces acteurs prennent part et analyserons enfin la structure de marché découlant de cette organisation. 


\section{Motivations des principaux acteurs}

\section{Les institutions muséales}

3 Faire itinérer ses expositions à l'international n'est pas une activité nouvelle pour les institutions muséales. Celle-ci se développe dès le milieu du XIXe siècle, d'abord à des fins d'éducation du public éloigné du musée, puis à des fins de rayonnement à l'international pendant l'entre-deux guerres. C'est à la fin des années 1990 et au début des années 2000, alors que les institutions muséales entrent progressivement dans un nouvel âge caractérisé par de nouveaux modes de gouvernance, une diversification de leurs ressources propres et une plus grande autonomie ${ }^{2}$, que cette possibilité de réaliser des économies grâce à l'itinérance est considérée avec de plus en plus d'intérêt. La crise économique de 2008, qui a provoqué la stagnation, voire la diminution, des principaux financements - publics comme privés - des musées dans le monde renforce encore davantage l'attention portée à cette activité. Aujourd'hui, développer l'itinérance de leurs expositions apparaît aux institutions muséales comme un moyen de répondre aux diverses injonctions qu'elles reçoivent en termes d'autofinancement, de rayonnement, de renouvellement de leur programmation ou encore de fréquentation, dans un contexte où s'intensifie la mise en compétition internationale des institutions.

4 Le raisonnement sous-jacent est simple : en faisant itinérer une exposition, on espère amortir les coûts de production de cette dernière sur un plus large bassin de visiteurs et/ ou d'institutions, et éventuellement parvenir à terme à dégager quelques bénéfices. Il est alors nécessaire de concevoir une exposition qui soit d'emblée la plus adaptée à l'itinérance et à ses contraintes, en termes d'expôts, de contenus et de scénographie. Faire voyager des œuvres nécessite par exemple la mise en place de mesures de conservation préventive tandis que la réalisation de reproductions requiert une négociation adaptée des droits de reproduction au préalable. Si une institution souhaite développer l'itinérance de ses expositions à des fins entre autres économiques, elle se doit d'intégrer parfaitement ces contraintes sous peine d'avoir à dépenser davantage pour permettre une itinérance que celle-ci ne sera en mesure de lui faire gagner. Par ailleurs, il s'agit de développer diverses compétences en termes de promotion, de négociation et de logistique. Faire itinérer des expositions nécessite en somme des moyens matériels, humains, organisationnels et financiers importants, et ne concerne donc que les institutions qui, conscientes de l'attention que cette activité requiert, sont capables de mobiliser les moyens nécessaires.

5 Une institution muséale peut aussi souhaiter accueillir une exposition itinérante internationale. Cela lui permet de renouveler sa programmation et éventuellement son public en proposant des expositions différant de sa ligne éditoriale traditionnelle et qu'elle n'aurait pas nécessairement eu le temps ni les moyens de concevoir. Ces possibilités suscitent bien entendu l'intérêt des musées dans un contexte où l'on exige des institutions une programmation fournie et rythmée, et où leurs résultats en termes de fréquentation conditionnent bien souvent leurs subventions. Accueillir des expositions itinérantes présuppose aussi un certain nombre de conditions. Il faut idéalement disposer d'un espace d'exposition peu contraignant, et avoir développé en interne une culture favorable à cette politique d'accueil. Il peut être en effet difficile pour les équipes en place d'avoir à travailler sur une exposition dont elles ne sont pas les conceptrices. 


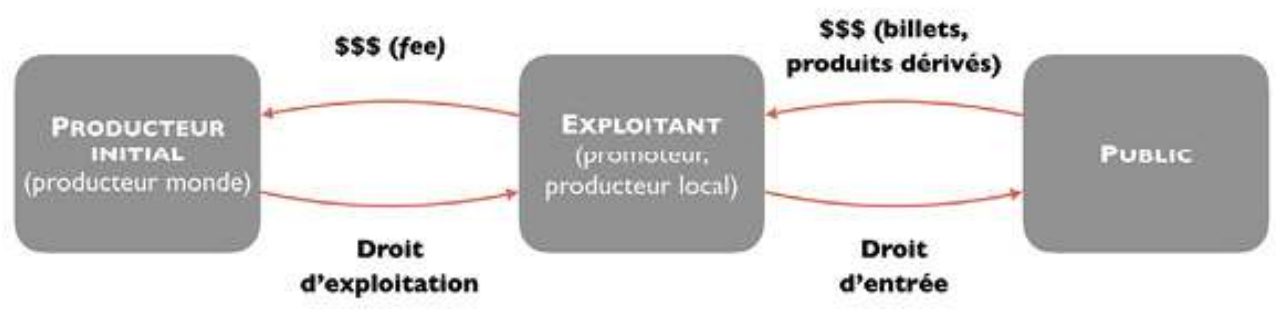

(c) Méliné Kéloglanian

\section{Les acteurs privés}

6 C'est dans le dernier quart du XXe siècle que des acteurs privés tels que Body Worlds ou Premier Exhibitions ont commencé à s'intéresser aux expositions itinérantes internationales. Le succès rencontré a ensuite encouragé un certain nombre de vocations. Tout autour du monde, une centaine d'entreprises privées produisent ou promeuvent aujourd'hui des expositions itinérantes d'envergure internationale. Les principaux acteurs se situent essentiellement en Amérique du Nord, en Europe et en Australie. Parmi les structures les plus importantes, outre les pionniers déjà cités, se trouvent entre autres les entreprises états-uniennes Imagine Exhibitions - fondée en 2009 par Tom Zaller, ancien de chez Premier Exhibitions - et EDG, fondée en 2006 par Amy Noble Seitz. En Europe, on compte notamment la société allemande SC Exhibitions qui a fait une entrée triomphante dans le secteur avec sa première production Toutankhamon: son tombeau, ses trésors et ses millions de visiteurs, et l'entreprise autrichienne EMS Entertainment. L'entreprise australienne Grande Exhibitions a quant à elle développé un système de projection immersif qui lui a permis de s'installer comme l'un des plus importants acteurs du secteur.

7 La plupart des producteurs privés qui ont rejoint ce domaine y ont vu un moyen de diversifier judicieusement leurs activités et leurs revenus et ont souvent préalablement développé un intérêt réel pour le média exposition en tant que vecteur de savoirs et d'émotions. Certains proviennent du monde du spectacle et du show business et sont habitués à investir dans des projets coûteux et risqués. Ils sont familiers des ressorts du divertissement et maîtrisent la logistique des tournées. D'autres sont issus du monde des musées - conservateurs, mais aussi transporteurs ou encore scénographes -, d'autres encore viennent du monde des salons et des foires internationales. Tous ont su mettre à profit leurs connaissances et compétences développées dans le cadre de leurs activités précédentes.

8 Lorsqu'il s'agit de recevoir une exposition itinérante internationale, nous recensons là encore des structures privées différentes des institutions muséales et susceptibles d'accueillir et d'organiser la présentation d'une telle exposition au public. Parmi elles, on compte notamment des centres commerciaux, mais aussi des aéroports, des parcs d'attraction ou encore des lobbys d'hôtels. Le centre commercial Evry 2 à Evry a reçu par exemple plusieurs expositions produites par Universcience, tandis que le parc Pacific National Exhibition à Vancouver recevait en 2016 l'exposition Angry BirdsTM Universe de 
l'américain Imagine Exhibition. Cette dernière société a par ailleurs présenté deux de ses expositions dans l'hôtel-casino The Venetian à Macao en 2012. Les expositions itinérantes permettent à ces structures de diversifier leur offre d'activités, et de donner ainsi à leurs visiteurs de nouvelles raisons d'initier, de prolonger ou tout simplement d'apprécier leur visite de ces lieux. Pour les centres commerciaux, cette programmation s'inscrit dans la tendance du retailtainment, stratégie visant à proposer aux visiteurs une expérience d'achat mêlant shopping et divertissement.

Quelles que soient les motivations qui incitent les institutions muséales et les acteurs privés à produire ou à accueillir des expositions itinérantes internationales, celles-ci ont soutenu la mise en place d'une offre et d'une demande, et donc d'un marché sur lequel interagissent des acteurs extrêmement variés. Ce marché prend forme notamment à travers les différentes plateformes et organisations dédiées à la rencontre de ces acteurs et au recensement des expositions itinérantes disponibles tels que les Touring Exhibitions Meetings, la base Informal Learning Experiences ou encore la base Extra du réseau Ecsite ${ }^{3}$. Pour mieux comprendre en quoi consistent les relations qu'entretiennent ces différents acteurs, il nous importe à présent d'étudier la filière de l'exposition itinérante internationale.

Répartition des acteurs privés dans le monde. Les acteurs ici dénombrés sont ceux que nous avons pu répertorier dans le cadre de nos recherches, en essayant de retrouver l'origine et les différentes étapes des tournées des expositions itinérantes internationales sur le marché en 2017, ainsi qu'en nous basant sur les listes des structures ayant participé à diverses conférences du secteur, telles que les Touring Exhibitions Meeting.

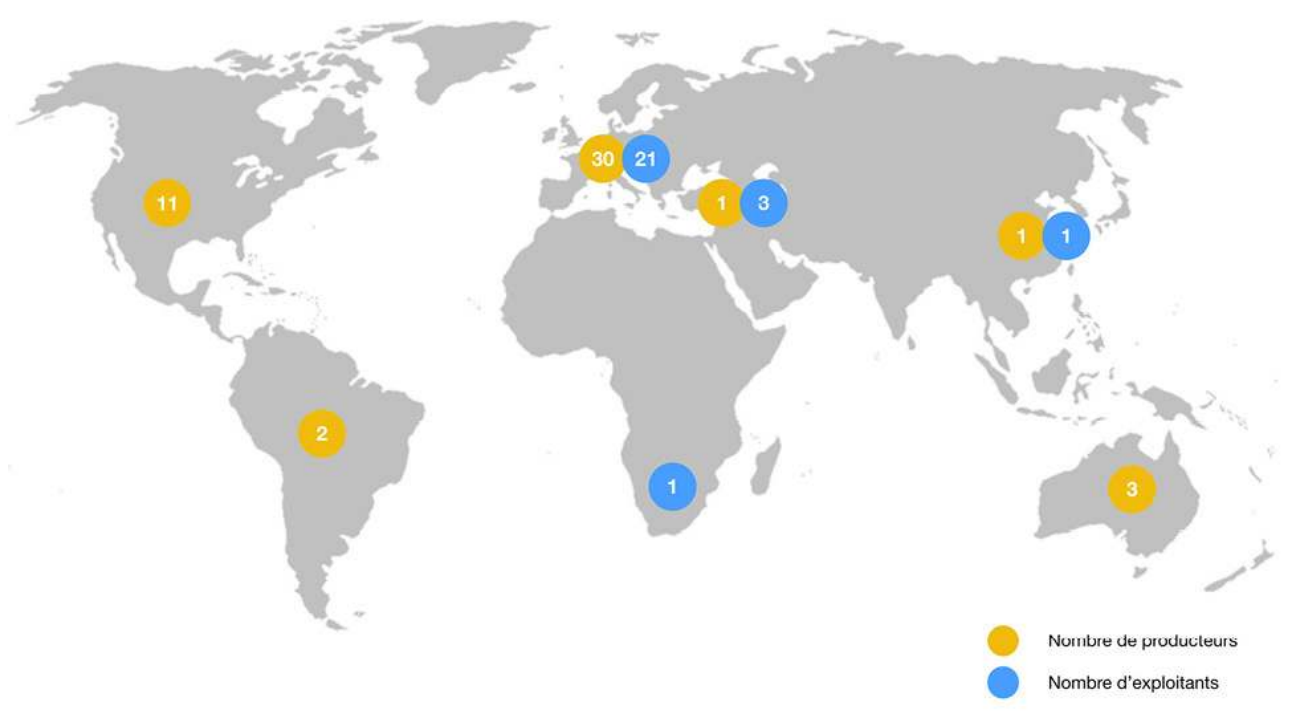

(C) Méliné Kéloglanian

\section{La filière de l'exposition itinérante internationale}

\section{Étapes et fonctionnement}

Plusieurs étapes sont nécessaires pour qu'un visiteur puisse effectivement visiter une exposition itinérante internationale. L'ensemble de ces étapes constitue une filière au sens économique du terme, soit "une succession d'opérations effectuées par des unités ayant des activités diverses, [...] liées entre elles par des opérations d'achats et de ventes"4. 
11 La première étape consiste en la production de l'exposition, elle est assurée par un producteur "initial" appelé aussi producteur monde. Celui-ci prend en charge la conception, le développement et la production initiale de l'exposition et en assume les coûts. Ce producteur octroie ensuite à un exploitant le droit de présenter son exposition sur un espace donné pour une durée déterminée, en échange d'une compensation financière appelée fee : un fee simple qui consiste en un forfait calculé sur la base de la durée de la cession de droit d'exploitation (forfait à la semaine, au mois), ou un fee double constitué de ce même type de forfait auquel s'ajoute un pourcentage des recettes de billetterie touchées par l'exploitant. Ce dernier assure alors la production locale de l'exposition, c'est-à-dire qu'il prend en charge son adaptation si nécessaire, sa promotion auprès d'un bassin de visiteurs, et sa présentation - ouverture d'un espace au public, mise en place d'une billetterie et d'une sécurité adéquate... L'exploitant est parfois désigné par les termes de promoteur ou de producteur local. Il assume les coûts de cette production locale et octroie ensuite aux visiteurs un droit d'entrée au sein de l'exposition, le plus souvent contre rémunération.

Ces étapes demeurent quelles que soient l'exposition itinérante considérée et la nature des acteurs prenant part à sa production et à sa présentation. Nous pouvons néanmoins observer des différences dans la manière de les mettre en œuvre suivant l'organisation de chaque producteur. Certains acteurs privés misent par exemple sur la polyvalence de leurs employés : une personne peut être à la fois responsable des ventes sur une certaine zone géographique, chargée de la production et de la logistique de tournée d'un projet et consultée pour la stratégie marketing d'un autre. Il en résulte des structures réduites à une équipe nucléaire, autour de laquelle gravite un ensemble d'employés non permanents sollicités au gré des projets. Le producteur gagne ainsi en compétitivité en allégeant au maximum ses coûts fixes et notamment ses charges de personnel ; il adapte sa force de travail au sujet des expositions, au nombre de projets en cours et peut alors proposer des prix en phase avec la réalité de sa production. Cela est plus difficile pour les institutions pour lesquelles les expositions proposées à l'itinérance ont suscité l'implication - souvent difficilement évaluable de façon objective - de nombreux services.

13 Concernant la phase d'exploitation des expositions itinérantes, les acteurs privés sont là aussi susceptibles de présenter une organisation et un modèle de rentabilité différents de ceux des musées. En effet, chaque exposition nécessitant des conditions particulières en termes de surface, mais aussi de hauteur sous plafond, d'hygrométrie et de sécurité, il incombe à l'exploitant qui en a acheté les droits de trouver un espace, appelé venue, qui soit à la fois disponible et conforme au cahier des charges. Deux cas de figure sont envisageables : soit l'exploitant dispose d'un lieu dont il a la charge et choisit alors des expositions pour lesquelles il serait adapté, soit l'exploitant ne dispose d'aucun lieu et doit alors en louer un. Les institutions culturelles telles que les musées se trouvent généralement dans le premier cas de figure, ainsi que certaines structures privées évoquées précédemment - centres commerciaux ou parcs d'attraction. Dans le second cas de figure, par exemple lorsque l'exploitant est une société de promotion et de production spécialisée, l'exploitant doit trouver un venue (parcs d'expositions, grandes halles, palais des congrès) et en assumer le prix de location.

Lorsque la Cité de la musique-Philharmonie de Paris présentait en 2015 l'exposition David Bowie is produite par le Victoria and Albert Museum de Londres, elle se trouvait dans le premier cas de figure. En échange d'un fee, elle a obtenu le droit de présenter cette exposition au sein de son propre espace d'exposition temporaire pour une durée limitée. 
Suivant l'autre cas de figure à présent, lorsqu'en 2014, l'exposition Star WarsTM Identities initialement produite par l'entreprise montréalaise X3 Productions - était présentée à la Sucrière à Lyon, elle n'était pas exploitée par cet établissement lyonnais mais par la société Les Productions Adonis. Ce promoteur ne disposant pas d'espace d'exposition a dû louer un venue pour présenter cette exposition dont il avait acheté les droits.

Brochure présentant l'exposition itinérante T-Rex, the killer question produite par le muséum d'Histoire naturelle de Londres, en coproduction avec la société japonaise Kokoro, productrice d'animatronics.

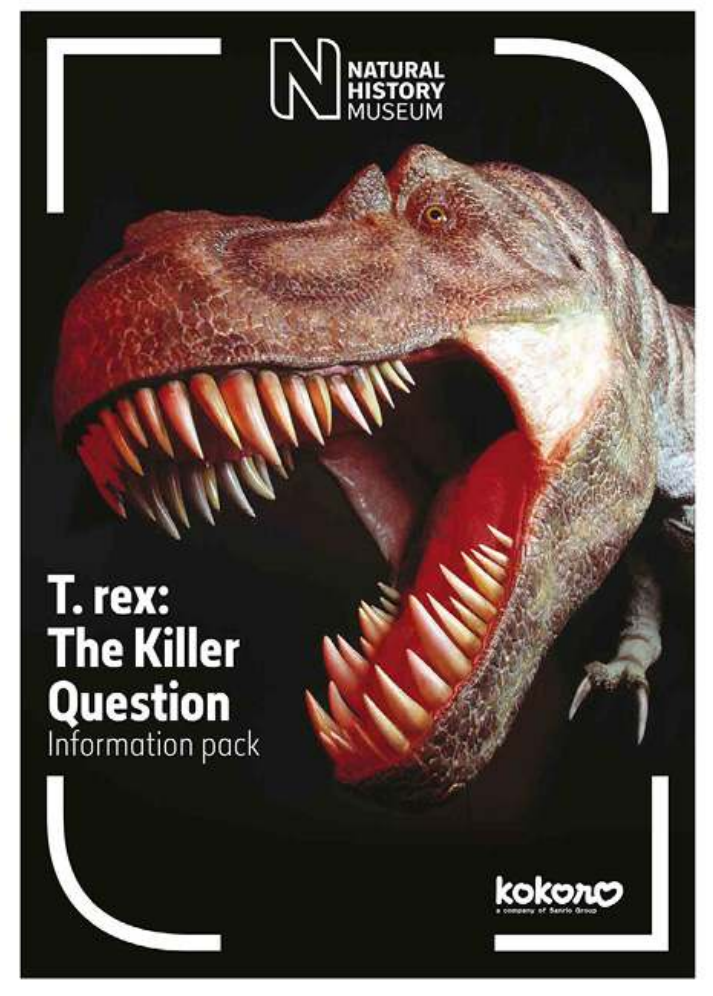

(C) DR

\section{La gestion du risque}

Lorsqu'un producteur initial s'engage dans la production d'une exposition itinérante internationale, il prend le risque de voir ses investissements ne jamais être rentabilisés dans le cas où il n'y aurait pas suffisamment d'exploitants intéressés. Il en est de même pour l'exploitant qui, lorsqu'il décide de présenter une exposition, n'a aucune certitude quant au nombre de visiteurs qu'il parviendra à mobiliser. Chaque production étant bien différente des autres, il est impossible de garantir indubitablement le succès d'une exposition en se basant par exemple sur une exposition qui aurait déjà été réalisée. Nous nous trouvons ici dans le cas d'une économie dite "de prototypes", à l'instar des industries créatives telles que celles du cinéma, du livre ou encore du spectacle vivant. Les acteurs font néanmoins usage de certaines pratiques afin de réduire ces risques. Le producteur initial peut par exemple présenter un projet d'exposition à différents exploitants et leur proposer de la réserver en échange d'un acompte avant même que celle-ci ne soit créée. Ce n'est que lorsqu'il a rassemblé suffisamment d'acomptes que le producteur se lance alors dans la réalisation de ce projet. Le producteur initial réduit 
ainsi le risque lié à la production de l'exposition en en transférant une partie à l'exploitant. Nous parlons parfois improprement de "préventes" pour désigner cette pratique. Celle-ci nécessite en tout cas de s'appuyer sur des outils marketing et promotionnels appropriés car il s'agit d'être suffisamment convaincant pour donner envie d'acheter une exposition qui n'existe qu'à l'état de projet.

Plutôt que de transférer une partie du risque, le producteur peut aussi décider de partager ce risque en faisant appel à différents investisseurs ou en ayant recours à la coproduction, comme le font les institutions muséales. Après avoir été présentée au sein des différentes institutions collaboratrices, l'exposition coproduite peut ensuite poursuivre son itinérance en dehors du cercle des coproducteurs, qui se répartissent alors les éventuels bénéfices. Cette solution permet aussi aux institutions partenaires de réaliser des projets de plus grande ampleur. Il est aussi possible de mieux gérer le risque non pas en le réduisant mais en le diversifiant. À l'instar des investisseurs financiers qui cherchent à diversifier correctement leur portefeuille de titres pour ne pas concentrer leurs risques sur un seul type d'actif, les producteurs d'expositions itinérantes peuvent décider de produire non pas une, mais plusieurs expositions, en pariant sur différents projets dont les échecs ne seraient pas corrélés. Ces expositions constituent le catalogue, appelé aussi le portefeuille ou le portfolio d'un producteur.

17 Les statuts juridiques des acteurs privés leur confèrent une capacité à agir rapidement, ce qui est avantageux pour la gestion de ces différents risques. À la différence des institutions publiques, ils ne sont pas soumis aux règles de la commande publique. Cela peut leur permettre de produire des expositions itinérantes plus rapidement et d'être plus réactifs pour le suivi logistique et la mise en place des itinérances. Les acteurs privés peuvent par ailleurs développer des activités ouvertement lucratives et garantir une entrée de recettes, en développant par exemple des produits dérivés - il s'agit néanmoins d'une activité difficile à mettre en place et qui nécessite une réelle expertise. Afin de bénéficier de certains des avantages que présentent les statuts juridiques d'une entreprise privée, certains musées ont pris le parti de créer une filiale indépendante pour gérer l'itinérance de leurs expositions. Cela leur permet aussi de préserver l'institution muséale mère des prises de risques inhérentes au développement de cette activité. C'est le cas du centre de science Heureka à Vantaa en Finlande qui a créé Heureka Overseas Productions LTD pour gérer ses activités d'itinérance et plus récemment de conseils.

Outre l'importance du risque et de sa bonne gestion au sein du secteur de l'exposition itinérante internationale, l'organisation de cette filière laisse aussi deviner la structure de son marché. Il convient alors de décrypter cette structure de marché, afin de comprendre comment les acteurs de ce secteur, dont nous connaissons à présent les motivations et les relations qu'ils sont susceptibles d'entretenir, peuvent s'y positionner. 
Affiche de l'exposition Quoi de neuf au Moyen Âge ? produite par Universcience. Il s'agit d'une exposition itinérante produite par une institution muséale qui a parfaitement intégré les contraintes liées à l'itinérance et a choisi pour cette exposition de proposer différents modules indépendants construits à l'aide de structures autoportantes.

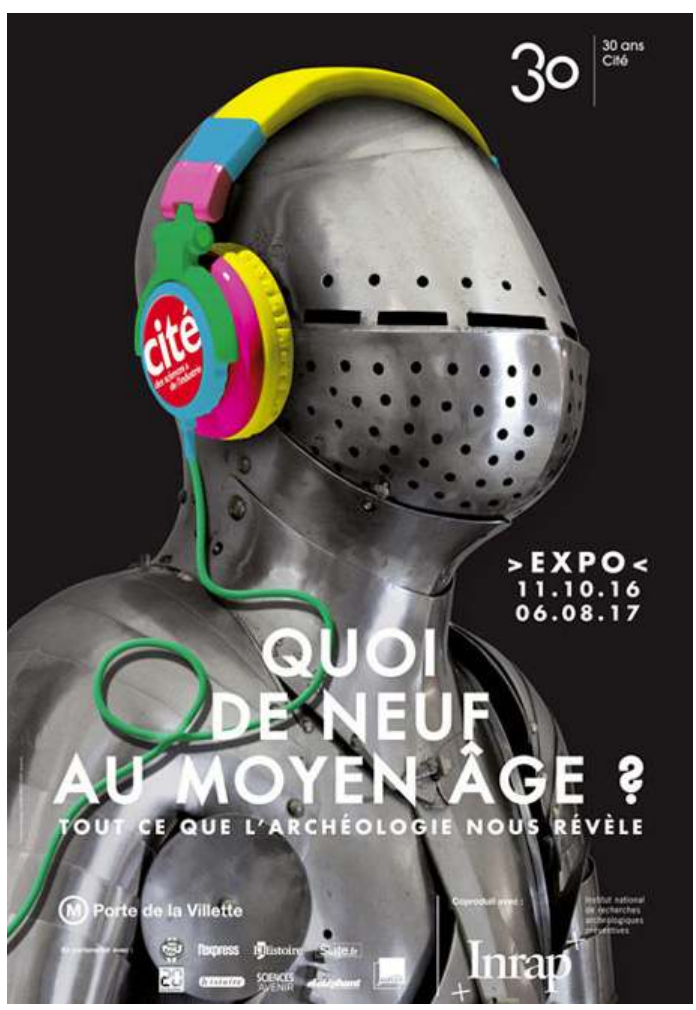

(c) Universcience

\section{Le marché de l'exposition itinérante internationale}

La filière de l'exposition itinérante internationale s'articule autour de deux temps forts : les producteurs d'expositions itinérantes internationales proposent tout d'abord aux exploitants un ensemble de productions - premier temps -, que ces derniers présentent ensuite aux visiteurs - second temps. Nous avons affaire dans ce premier temps à des relations d'entreprises à entreprises, nous parlons en économie de relation $B$-to-B pour business-to-business, puis dans ce second temps à des relations d'entreprises à consommateurs, on parle de relation $B-$ to- $C$ pour business-to-consumer. L'ensemble du secteur repose donc sur une logique $B$-to-B-to- $C$ : les actions entreprises par les producteurs initiaux (B1) envers les exploitants (B2) ne se justifient que - et sont aussi conditionnées dans une certaine mesure - par le fait qu'in fine un visiteur (C), destinataire final de l'exposition, vient effectivement la visiter. Lorsqu'il envisage de produire une nouvelle exposition, un producteur initial doit bien connaitre ces deux niveaux de marché afin de s'y positionner correctement.

\section{La partie B-to-B du marché}

Pour mieux appréhender cette partie haute de la filière, le producteur initial peut mettre en place des activités de veille, mener des études de marché, de faisabilité ou encore 
tester ses idées auprès d'exploitants. Chaque producteur initial développe ainsi sa propre compréhension du marché et met au point la ou les segmentations qui lui semblent les plus appropriées. Deux lectures sont le plus souvent utilisées : par typologie d'exploitant et par zone géographique. Les exigences des exploitants vis-à-vis des caractéristiques de l'exposition ne sont en effet pas les mêmes suivant s'il s'agit d'un musée, d'un promoteur privé ou d'un centre commercial. L'organisation des centres commerciaux exige par exemple des expositions itinérantes nécessitant peu de personnel de surveillance, montables et démontables extrêmement rapidement. Sur le plan géographique, il existe des différences aussi bien en termes d'équipements que d'ap-

proches : tandis que nous aurons par exemple davantage affaire à des musées et des centres de sciences en Amérique du Nord, on privilégiera des relations avec des promoteurs en Europe de l'Est ; on favorisera les approches relevant de l'edutainment des termes anglais education et entertainment - dites aussi ludo-éducatives pour les institutions nord-américaines ou la présence d'œuvres originales pour les expositions à destination de la Chine.

L'exposition Hunger Games produite par Imagine Exhibitions est un exemple d'exposition s'appuyant sur l'exploitation d'une franchise.

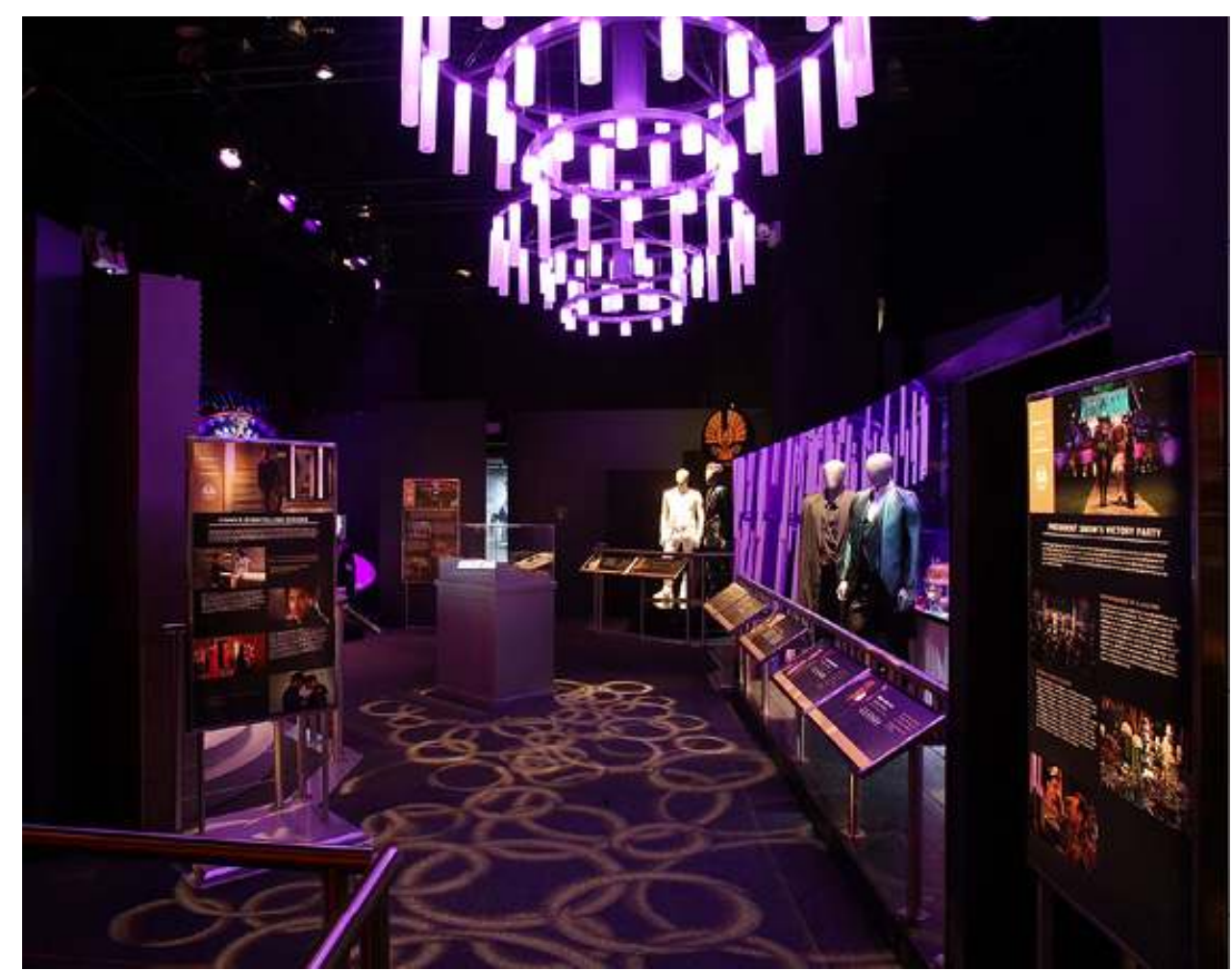

(C) Imagine Exhibitions

\section{La partie B-to-C du marché}

Pour être en phase avec le public recherché, il est nécessaire de savoir favoriser une approche ou un sujet plutôt qu'un autre. Ceci nécessite au préalable de s'être intéressé aux attentes du destinataire final de l'exposition, ce consumer en bout de filière, le visiteur. Compte-tenu des risques précédemment cités, il est sécurisant de traiter de 
sujets et de mobiliser des approches susceptibles de fonctionner dans un maximum de situations ; des sujets qui soient des "valeurs sûres", capables d'attirer du public tout autour du globe, et donc in fine d'intéresser les exploitants, institutions muséales ou acteurs privés, quelles que soient leurs origines géographiques.

Parmi ces sujets, on compte notamment les dinosaures, les secrets du corps humain ou encore le naufrage du Titanic. Le succès rencontré par les expositions traitant de ces thématiques en a fait des incontournables des catalogues d'expositions des producteurs. On a affaire à de véritables expositions blockbusters, ces superproductions qui n'ont d'autres choix que de rencontrer le succès ${ }^{5}$.

Les franchises, telles que Jurassic World, Hunger Games ou encore Harry Potter, sont elles aussi un réservoir de "valeurs sûres", à en juger par la recrudescence d'expositions s'appuyant sur ces univers. Tandis que le producteur privé Imagine Exhibitions se propose de démystifier les lois de la physique en s'appuyant sur les trajectoires des oiseaux du jeu Angry Birds, la Smithsonian Institution invite à découvrir le processus de création de costumes de cinéma en prenant exemple sur la saga Star Wars.

Le poids de ces sujets dans l'offre d'exposition itinérante internationale soulève la question de la mise en place d'une culture mainstream universelle dans le but de répondre à des logiques de marché et de diminution du risque. Aujourd'hui, compte tenu de la répartition géographique des acteurs et principalement des producteurs initiaux dans le monde, la provenance de cette culture mainstream interpelle, questionne l'hégémonie d'un modèle américain d'entertainment de masse ${ }^{6}$ et met en lumière les enjeux géopolitiques - qu'il conviendrait d'étudier plus amplement par ailleurs - que revêt l'activité d'itinérance.

\section{Le cas particulier de la France}

26 Le réseau d'infrastructures, la culture d'accueil de ces infrastructures, ainsi que les spécificités culturelles d'une zone géographique sont des éléments clés pour comprendre les deux niveaux de marché constituant le secteur de l'exposition itinérante internationale sur un territoire. Différentes échelles d'étude sont néanmoins envisageables pour mieux appréhender celui-ci. À l'échelle de l'Europe, la France apparaît comme un cas particulier. Elle se distingue en effet par une absence de producteur privé spécialisé dans cette activité et un faible nombre de promoteurs comparé à des pays voisins tels que l'Allemagne ou l'Italie. Le rattachement historique des affaires culturelles au service public peut être l'une des explications de ce cas particulier et de la réticence perceptible à envisager l'intervention d'acteurs privés. Il faut rappeler aussi que les expositions itinérantes internationales nécessitent des infrastructures particulières, des moyens financiers relativement conséquents, ainsi qu'une zone de chalandise importante que peu d'institutions ou centres sont en mesure d'avoir à disposition en France. Le manque de telles infrastructures et l'armature urbaine de la France expliquent sans doute en partie cette situation particulière. Néanmoins, il existe à présent quelques promoteurs privés, tels que les Productions Adonis ou Encore Productions. La prochaine étape pour le territoire français est donc vraisemblablement le développement de producteurs privés d'expositions itinérantes internationales ; cela marquerait un tournant significatif dans l'essor du versant privé de ce marché en France. 


\section{Conclusion}

Lorsque l'on considère le secteur de l'exposition itinérante internationale dans sa globalité, on constate que les institutions muséales prennent place sur un marché reposant sur la rencontre d'une offre et d'une demande composites, soutenues par les motivations d'acteurs de nature très diverse - qu'il s'agisse d'anciens producteurs de show-business, de scénographes, de centres commerciaux ou de parcs d'expositions. Ces structures, productrices ou exploitantes, parfois simples venues, interviennent à différents stades d'une filière, au sein de laquelle le risque lié à la dimension prototypique des expositions itinérantes et sa bonne gestion jouent un rôle essentiel. Les temps forts de cette filière permettent par ailleurs de prendre conscience de la structure générale du marché qui consiste en un marché B-to-B-to-C. Cette structure impose aux différents acteurs de connaître alors deux niveaux de marché pour s'y positionner correctement.

Compte tenu de l'intérêt des institutions muséales pour l'exposition itinérante internationale, et des enjeux que revêt ce produit culturel et les logiques de fonctionnement de son marché, il semble plus que jamais essentiel de continuer à approfondir notre connaissance de ce secteur et d'en suivre de près les évolutions. Ainsi, les institutions muséales n'auront que plus de chance et de facilité à satisfaire aux exigences qu'on leur impose en termes d'autofinancement, de rayonnement, de renouvellement de leur programmation ou encore de fréquentation, en continuant d'assurer leurs missions et de préserver les spécificités de leur discours.

Amsellem, R. International Touring Exhibitions: Toward a Profitable Business Model for Exhibitions? The Journal of Arts Management, Law, and Society, vol. 43, n¹, 2013, pp. 36-57.

Armstrong, G. et Kotler, P. Principes de marketing. Paris : Pearson Education, 2010, 457 p. Buck, R.-A. et Gilmore, J.-A. On the road again: developing and managing traveling exhibitions. Washington: American Association of Museums, 2003, 158 p.

Busson, A. et Evrard, Y. Le management des industries culturelles et créatives. Paris : Vuibert, 2015, $294 \mathrm{p}$.

Chaumier, S. (dir.) Expoland, ce que le parc fait au musée; ambivalence des formes de l'exposition. Paris : Complicités, Coll. Muséo-Expographie, 2011, 191 p.

Desvallées, A. et Mairesse, F. (dir.) Dictionnaire encyclopédique de muséologie. Paris : A. Colin, 201, $722 \mathrm{p}$.

Drouguet, N. Succès et revers des expositions-spectacles, Culture \& Musées, vol. 5, $n^{\circ} 1$, 2005, pp. 65-90.

Mairesse, F. et Rochelandet, F. Économie des arts et de la culture. Paris : Armand Colin, 2015, $271 \mathrm{p}$. 


\section{NOTES}

1. Nous ne considérerons ici que les expositions itinérantes destinées à des tournées internationales et revêtant un objectif avoué de rentabilité ou du moins, d'amortissement partiel des coûts de production de l'exposition en question.

2. Tobelem, J.-M. Le nouvel âge des musées : les institutions culturelles au défi de la gestion. Paris :A. Colin, 2010, p. 16.

3. Respectivement : http://touringexhibitions.org ; www.informallearning.com ; www.ecsite.eu/ activities-and-services/resources/extra.

4. "Le concept de filière fait [...] référence à l'idée qu'un produit, bien ou service, est mis à la disposition de son utilisateur final par une succession d'opérations effectuées par des unités ayant des activités diverses. Chaque filière constitue donc une chaîne d'activités qui se complètent, liées entre elles par des opérations d'achats et de ventes", in Monfort, J. À la recherche des filières de production, Économie et statistique, vol. 151, n¹, 1983, p. 3.

5. Les expositions Body Worlds par exemple, depuis la présentation du premier spécimen en 1995, ont reçu près de 44 millions de visiteurs à l'échelle mondiale, https://bodyworlds.com/ about/philosophy.

6. Martel, F. Mainstream : enquête sur cette culture qui plait à tout le monde. Paris : Flammarion, 2010, pp. 9-14.

\section{RÉSUMÉS}

L'organisation d'exposition itinérante internationale est l'une des réponses données par les institutions muséales

à diverses exigences, notamment d'autofinancement et de rayonnement. Cette contribution présente les motivations des différents partenaires ainsi convoqués et analyse les enjeux de ce produit culturel ainsi que les logiques de fonctionnement de son marché.

\section{INDEX}

Mots-clés : Société, inclusif, expérience

\section{AUTEUR}

\section{MÉLINÉ KÉLOGLANIAN}

Titulaire d'un master de recherche en muséologie et d'un MSc en Business Management (École du Louvre/ESSEC), chargée

de production à la Cité de la musique-Philharmonie de Paris 
meline.keloglanian@gmail.com 